\title{
Can chest CT improve sensitivity of COVID- 19 diagnosis in comparison to PCR? A meta-analysis study
}

Heba Mahmoud ${ }^{1}$, Mohamed Shehata Taha ${ }^{1}$, Anas Askoura ${ }^{1 *}$ (D), Mohammed Aleem ${ }^{1}$, Azza Omran² and Soha Aboelela ${ }^{3}$

\begin{abstract}
Background: In December 2019, SARS-CoV-2 was identified as the causative agent of pneumonia cases in China. This virus is spread by coughing or sneezing and can infect other persons by on contacting mucous membranes. SARS-Cov-2 most frequent serious manifestation is pneumonia. Chest computed tomography in COVID-19 patients usually shows ground-glass opacities that may be accompanied by consolidation lesions. Early diagnosis of the disease and rapid isolation of the patient is of great importance. So far, confirmation of COVID-19 infection is made by RT-PCR of nasopharyngeal or respiratory specimens. Recent research reported that the sensitivity of computed tomography in diagnosing COVID-19 is $98 \%$ while RT-PCR sensitivity is $71 \%$. Herein, we compare the sensitivity of both chest CT and RT-PCR in diagnosing COVID-19 at initial patient presentation through a meta-analysis study.
\end{abstract}

Main body: Using MEDLINE database a systematic literature search was conducted to identify relevant published studies within from November 2019 to April 2020. Only articles with full text were examined to determine eligibility and extract data by two reviewers. It was decided to include studies mentioning sensitivity of chest CT scan and sensitivity of RT-PCR and both done at the same time.

Results: Potentially relevant 15,300 studies were identified in our search in MEDLINE whose titles were quickly reviewed. Potentially eligible studies missing any of the forementioned inclusion criteria were excluded. This process left 7 eligible articles that fulfilled the inclusion criteria and were thus included in the meta-analysis and used for further analyses.

Conclusion: The meta-analysis study showed that chest CT may be beneficial in early detection of cases of COVID19. Imaging, in adjunct to clinical and laboratory findings, should be used for monitoring of disease course, until further evidence is available.

Keywords: SARS CoV-2, COVID-19, PCR, CHEST CT scan

\section{Background}

A new coronavirus was known, at the end of 2019, to cause a collection of pneumonia cases in Wuhan, China. As of the beginning of 2020, the virus spread rapidly resulting in a worldwide pandemic. The virus causing COVID-19 was named severe acute respiratory

\footnotetext{
* Correspondence: anas_askoura@med.asu.edu.eg

'Ear, Nose and Throat Department, Faculty of Medicine, Ain Shams University, Cairo, Egypt

Full list of author information is available at the end of the article
}

syndrome coronavirus 2 (SARS-CoV-2) [1]. The incubation period of SARS-CoV-2 infection is around 14 days; however, most of the cases show symptoms after 4 to 5 days of exposure [2-4]. Pneumonia is the most common extreme presentation of SARS-CoV-2 infection, manifesting by fever, dry cough, difficulty of breathing, and ground-glass infiltrates in radiological images [3, 5-7]. No unique clinical features can yet be used to differentiate COVID-19 from other pneumonic viral respiratory

\section{Springer Open}

() The Author(s). 2020 Open Access This article is licensed under a Creative Commons Attribution 4.0 International License, which permits use, sharing, adaptation, distribution and reproduction in any medium or format, as long as you give appropriate credit to the original author(s) and the source, provide a link to the Creative Commons licence, and indicate if changes were made. The images or other third party material in this article are included in the article's Creative Commons licence, unless indicated otherwise in a credit line to the material. If material is not included in the article's Creative Commons licence and your intended use is not permitted by statutory regulation or exceeds the permitted use, you will need to obtain permission directly from the copyright holder. To view a copy of this licence, visit http://creativecommons.org/licenses/by/4.0/. 
infections. CT imaging in COVID-19 patients commonly shows ground-glass opacities.

Sometimes accompanied by areas of consolidation, findings are consistent with viral pneumonia $[8,9]$. A recent case series reported that chest CT findings are more frequently to occur bilateral, have a peripheral distribution, and comprise mainly the lower lobes. Less common findings in COVID-19 patients include pleural thickening and pleural effusion [1].

In the absence of specific anti-viral drugs or vaccines for SARS-CoV-2, early detection of the disease and the instant isolation of an infected patient are crucial.

The National Health Commission of China states that the diagnosis of SARS-CoV-2 infection must be established by viral nucleic acid detection either by reversetranscription polymerase chain reaction (RT-PCR) or by sequencing of either respiratory or blood samples [10]. The total positive rate of PCR for throat swab samples is reported to be about 30 to $60 \%$ at the original presentation and this is mainly caused by confines regarding sample collection, sample transport, and kit performance [11].

Due to the lack of presence of RT-PCR in the current public health emergency, many COVID-19 patients are not identified quickly and are not receiving the appropriate treatment. In addition, as they do not know that they are infected and given the highly transmissible nature of the virus, they do bear a chance of infecting others. Chest CT may be a more accurate, available, realistic, and fast method for diagnosing and assessing suspected COVID-19 patients compared to RT-PCR. A recent study reported the sensitivity of CT to be $98 \%$ in diagnosing COVID-19 infection compared to RT-PCR which showed a sensitivity of $71 \%$ [11].

In this study, we aimed to compare the sensitivity of chest CT to the sensitivity of RT-PCR at the initial patient presentation through a meta-analysis study.

\section{Methods}

\section{Search for relevant studies}

We used the MEDLINE database (www.pubmed.com) to perform a systematic search of literature to find relevant studies that were published within the past 6 months (from November 2019 to April 2020). All appropriate articles were accessed in full text in order to determine the eligibility and extract the data by two reviewers.

We also scanned the references of the retrieved articles to find further studies that could have been missed in our initial search, the online searches were expanded. We agreed to only include studies that are reported or translated in English and that address chest CT scan sensitivity and RT-PCR sensitivity and both performed simultaneously (time of initial presentation). We excluded articles that missed one or more of the forementioned inclusion criteria, duplicated studies, or those outdated by newer ones and studies with provided data that cannot be extracted.

\section{Study selection and data abstraction}

From each article, the subsequent information was abstracted: type of the study (meta-analysis or randomized control trials, prospective, retrospective, and systematic review), sensitivity of chest CT scan, and sensitivity of RT-PCR.

\section{Statistical methods}

Statistical analysis was completed using the jamovi version 1.1 computer software (the jamovi project. jamovi version 1.1, 2019. Recovered from https:// www.jamovi.org).

Data were acceptable for conducting meta-analysis for the CT scan or RT-PCR sensitivity. Other accuracy indices could not be assessed.

Studies included in meta-analysis were tested for heterogeneity of the estimates by means of the Cochran $Q$ chi-squared test and $I$-squared statistic.

Publication bias was judged by analyzing funnel plots of the estimated effect size versus its standard error. To examine the asymmetry of funnel plots, the BeggMazumdar rank correlation and the Egger regression tests were used.

Effect sizes were pooled using random-effects maximum likelihood model. Pooled sensitivity is reported with $95 \%$ confidence limits and prediction interval.

\section{Results}

\section{Study identification and eligibility}

Our search recognized 15,300 potentially relevant studies in MEDLINE whose titles were quickly reviewed. Records after duplicates removed were 10,100 articles. There were 200 studies that were potentially eligible from them.

Out of the 200 studies, 150 were excluded as they lack one or more of the forementioned inclusion criteria or as they were outdated by other more recent ones. So, 50 studies were eligible for possible inclusion and were accessed in the full-text form. After going through the full length of the articles, 43 studies were excluded as some of them were essay studies while others did not mention the sensitivity of one or both researched items. This process left 7 original articles that fulfilled the inclusion criteria and were thus included in the meta-analysis and used for further analyses, PRISMA diagram [1].

\section{Analysis of included articles}

Among the 7 included articles, there were no randomized control studies. Only retrospective studies were 
found and used for additional analysis, Figs. 1, 2, 3 and 4 , and.

PRISMA diagram [1]

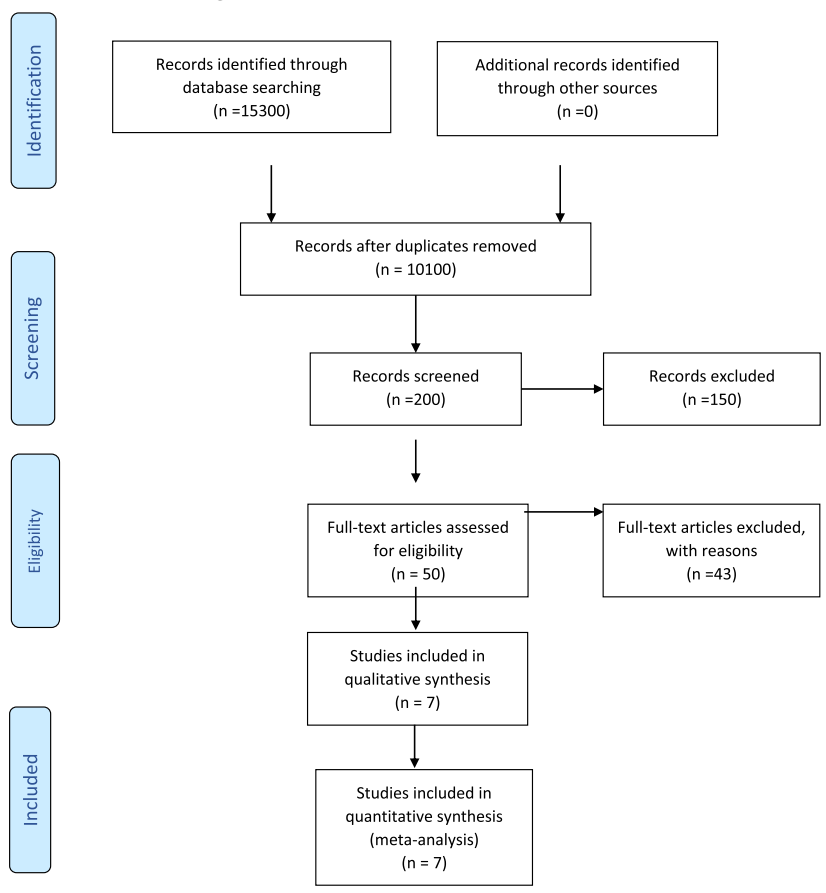

\section{Discussion}

The absence of specific anti-viral drugs or an anti-SARSCoV-2 vaccine necessitates the early diagnosis of COVID-19 to allow efficient disease control and treatment. Chest CT imaging could be a fast method, compared to RT-PCR, to early detect and assess COVID-19, particularly in the epidemic zone [11]. Clinicians are assessing more and more suspected patients, and so are radiologists who are correspondingly interpreting more and more chest CTs of patients suspected to have COVID-19. Clinical practice has shown that chest CT is an important component that aids in the diagnosis of patients with suspected COVID-19 infection. In fact, given the inadequacy of RT-PCR kits in some health facilities and the likelihood of occurrence of a false negative RT-PCR result for SARS-CoV-2, the National Health Commission of the People's Republic of China is encouraging diagnosis based on clinical findings in addition to chest CT findings alone [18].

In this meta-analysis, we aimed to investigate the sensitivity of chest CT scan versus the sensitivity of RTPCR in diagnosing COVID-19 infection at initial presentation.

Seven articles were eligible to this study. Forest plot test for sensitivity of initial CT scan indicates considerable heterogeneity across studies (Cochran $Q$ test $P$ value $<0.001, I$-squared $=94.2 \%)$, and the pooled sensitivity was $89 \%$ (95\% CI $=80$ to $97 \%$ ). Also funnel plot for sensitivity of initial CT scan shows no evidence of publication bias (Rosenthal fail-safe number $=37,526$, rank correlation test for funnel plot asymmetry $P$ value $=0.239$, regression test for funnel plot asymmetry $P$ value $=0.300$ ) (Figs. 1 and 2). On the other hand, the forest plot test for sensitivity of initial RT-PCR shows considerable heterogeneity across studies (Cochran $Q$ test $P$ value $<0.001, I$-squared $=98.2 \%)$ and pooled sensitivity $=70 \%$ (95\% CI $=50$ to $90 \%)$. Funnel plot for sensitivity of initial RT-PCR scan declares the possibility of publication bias (Rosenthal fail-safe number $=7902$, rank correlation test for funnel plot asymmetry $P$ value $=0.562$, regression test for funnel plot asymmetry $P$ value $=0.002)$ (Figs. 3 and 4 ).

Review and analysis of the data collected from the included 7 articles revealed that RT-PCR testing for viral nucleic acid plays a vital role in defining hospitalization and isolation for individual patients. However, it is less sensitive than chest CT (89\% versus 70\% respectively). Additionally, many external aspects can affect the result of RT-PCR test including sampling technique, specimen type (upper or lower respiratory tract), time of sampling (different period of disease development) [19], in addition to the performance of the used detection kits. Hence, RT-PCR test results must be interpreted with care as such.

Chest CT is a non-invasive, conventional imaging technique with high precision and speed. Based on recent published literature, nearly all patients with COVID-19 have distinctive CT features during the disease process $[8,11,12,20,21]$, e.g., variable degrees of ground-glass opacities that may be accompanied by crazy-paving sign, bilateral multifocal organizing pneumonia, and architectural alteration in a peripheral distribution. Liji Thomas stated that CT picked up nearly all cases that were detected using RT-PCR, and $75 \%$ of the cases that were initially missed by serological test. The authors reported that chest $\mathrm{CT}$ had a positive predictive value of $65 \%$ and a negative predictive value of $83 \%$. He also reported that in spite of a relatively high number of false positive, the priority in such an emergent situation is to identify the biggest number of cases as hastily as possible rather than absolute accuracy of diagnosis [14].

In a study performed by $\mathrm{Ai}, \mathrm{T}$ et al., $60 \%(34 / 57)$ of the cases had characteristic $\mathrm{CT}$ findings that are consistent with COVID-19 either prior to or parallel to the positive RT-PCR results. Also, nearly all patients (56/57) had initial chest CT findings before or within 6 days of the initial positive RT-PCR results showing that chest CT might be very valuable in the early detection of suspected cases [11].

It was proven that the standard diagnostic method being used is real-time polymerase chain reaction (RTPCR) for spotting viral nucleotides from specimens collected by oropharyngeal swab, nasopharyngeal swab, 


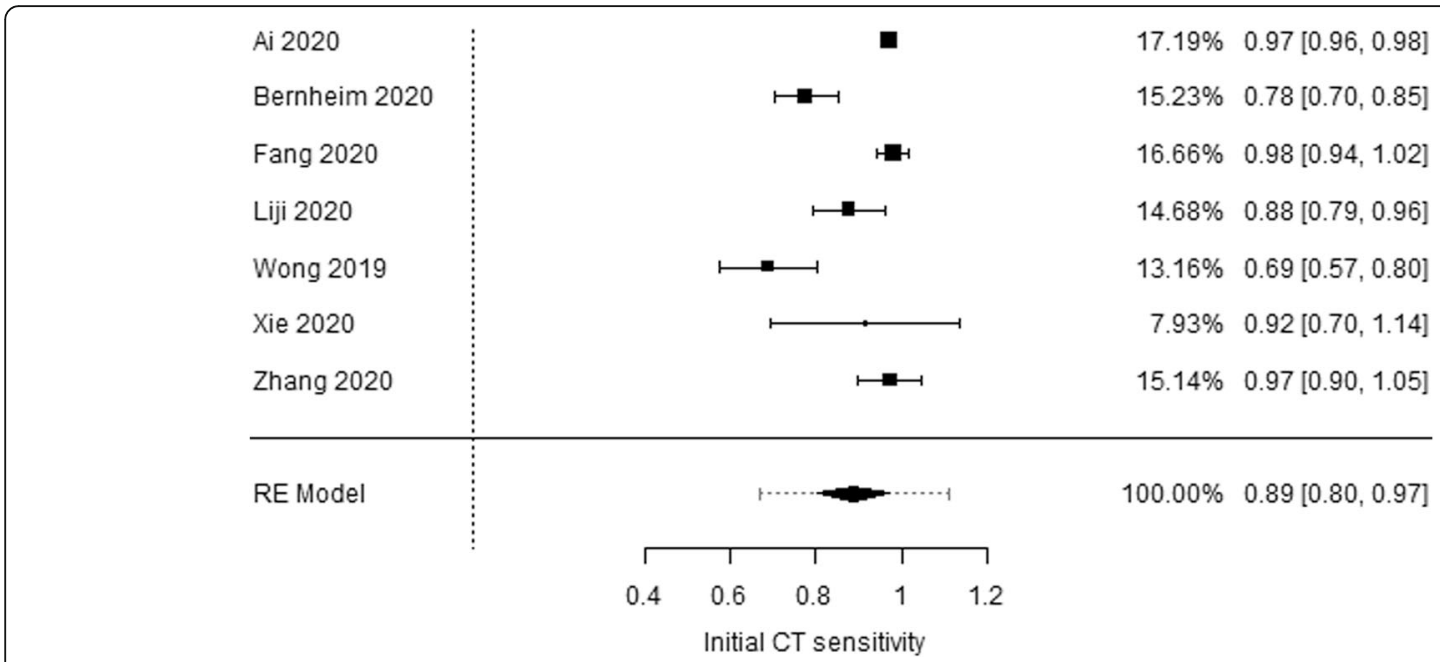

Fig. 1 Forest plot for sensitivity of initial CT scan. There is considerable heterogeneity across studies (Cochran $Q$ test $P$ value $<0.001$, 1 -squared $=$ 94.2\%). Pooled sensitivity $=89 \%$ (95\% Cl $=80$ to 97\%). Prediction interval is presented as a dotted extension up and down the $95 \%$ confidence limits of pooled effect size [11-17]

bronchoalveolar lavage, or tracheal aspirate [19]. Nonetheless, new reports have shown that RT-PCR has a sensitivity as small as $60-71 \%$ for detecting COVID-19 [11, 13, 20]. RT-PCR test results for COVID-19 may be false negative either due to laboratory errors or due to insufficient presence of viral material in the clinical sample $[13,16]$. Moreover, the current laboratory test still remains time consuming; moreover, a shortage in kit supply might not meet the growing needs of the infected population.
Such false negatives prolong quarantine attempts, require repeated testing and have the potential to overwhelm the current test kits and associated infrastructure supply [20]. By comparison, chest CT has demonstrated a sensitivity of about $56-98 \%$ in the identification of COVID-19 at preliminary presentation, and might be helpful in the correction of false negatives obtained from RT-PCR test during the early disease phase $[13,20]$.

Fang et al. stated that the sensitivity of chest CT in their analysis was superior to that of RT-PCR (98\%

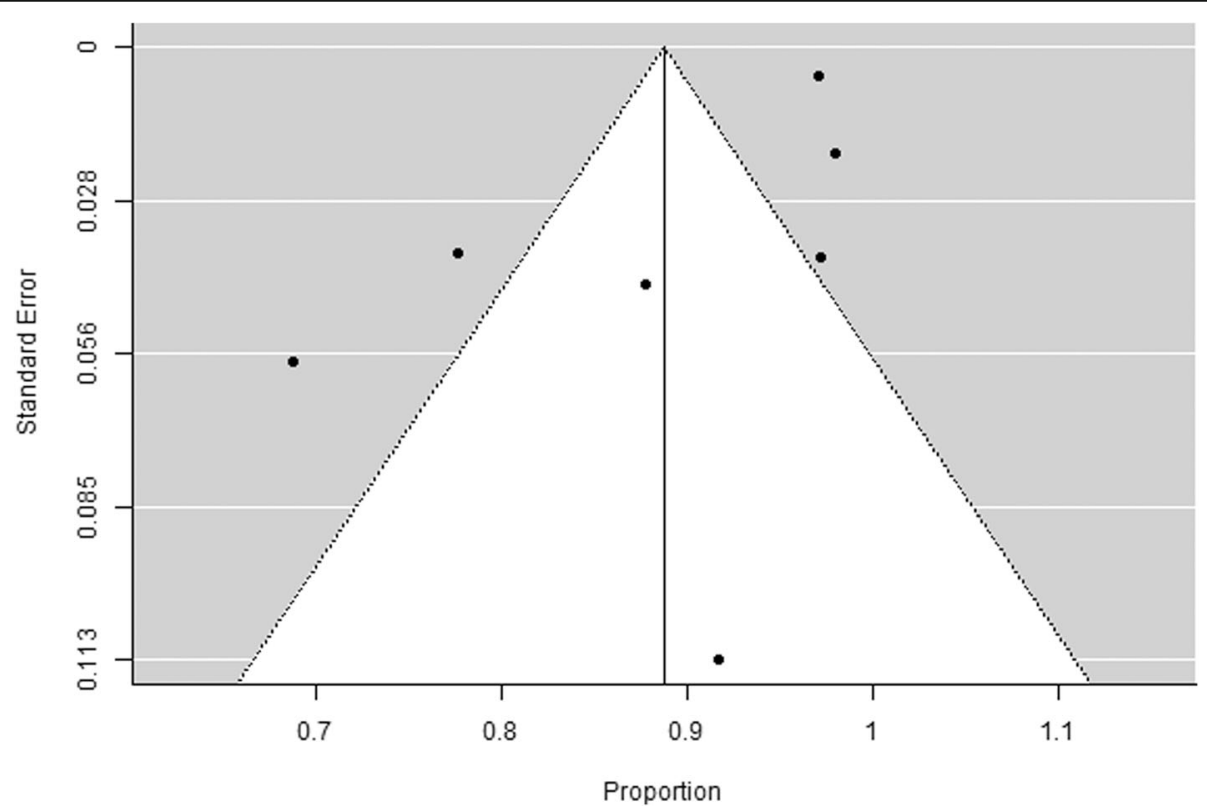

Fig. 2 Funnel plot for sensitivity of the initial CT scan. There is no evidence of publication bias (Rosenthal fail-safe number $=37,526$, rank correlation test for funnel plot asymmetry $P$ value $=0.239$, regression test for funnel plot asymmetry $P$ value $=0.300$ ) 


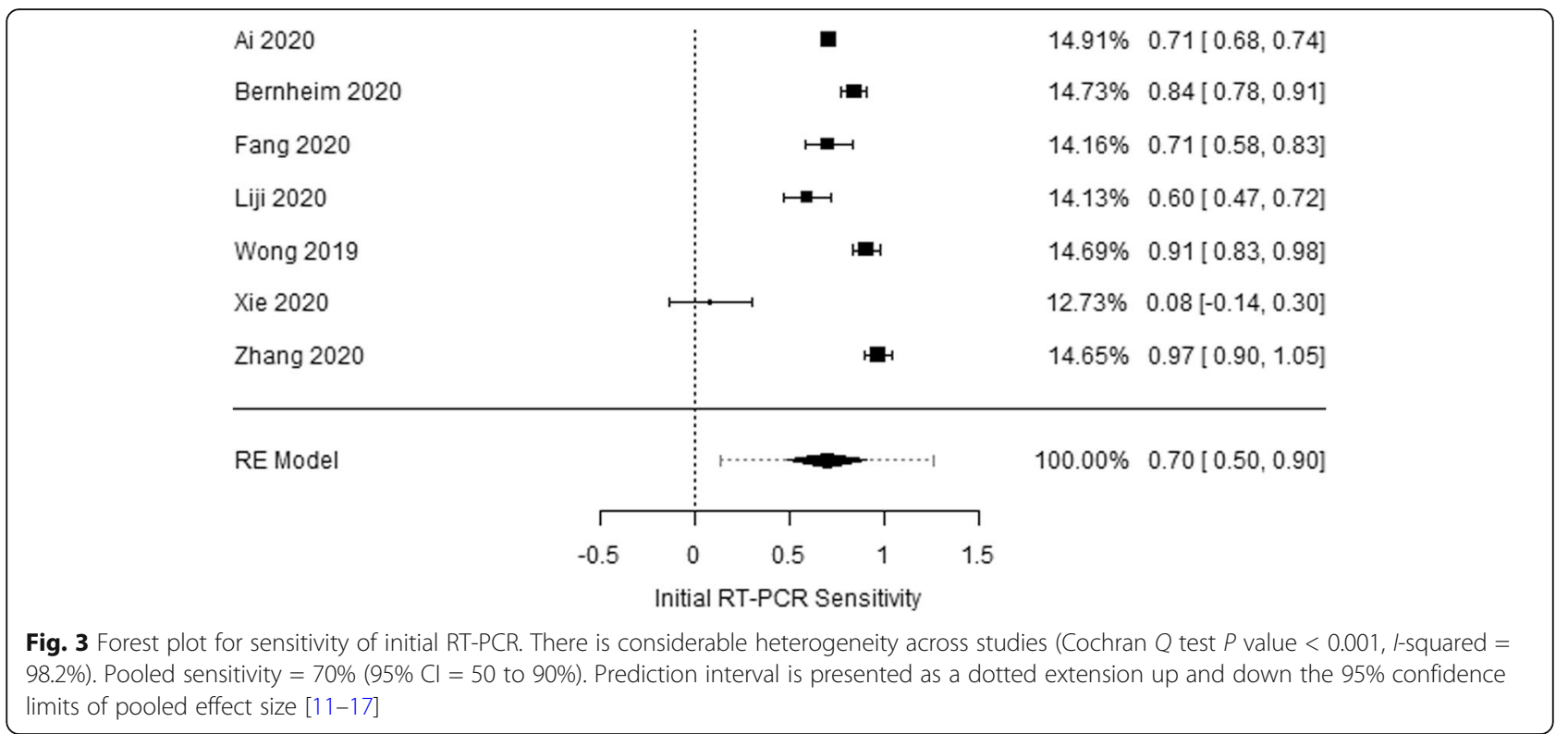

versus $71 \%$, respectively, $P<.001$ ). The authors attributed the reason for the low performance of RT-PCR to (1) the novelty of nucleic acid testing that still might need enhancement; (2) disparity in detection level from different suppliers; (3) patients who might have viral load; or (4) incorrect clinical sampling. They concluded that their results do support the use of chest CT in screening of COVD-19 patients with either clinical and/ or epidemiologic features indicative of COVID-19 infection especially when RT-PCR test is negative [13].
Recently, their result was supported by Xie who reported that $5 / 167(3 \%)$ patients had initially negative RT-PCR for COVID-19 in spite of chest CT findings characteristic of viral pneumonia at earlier presentation [16].

Shi and colleagues studied patients confirmed to have SARS-CoV-2 infection and reported abnormal chest CT findings even in asymptomatic patients. Obviously, their findings are important for the early clinical management of COVID-19 pneumonia patients. From an

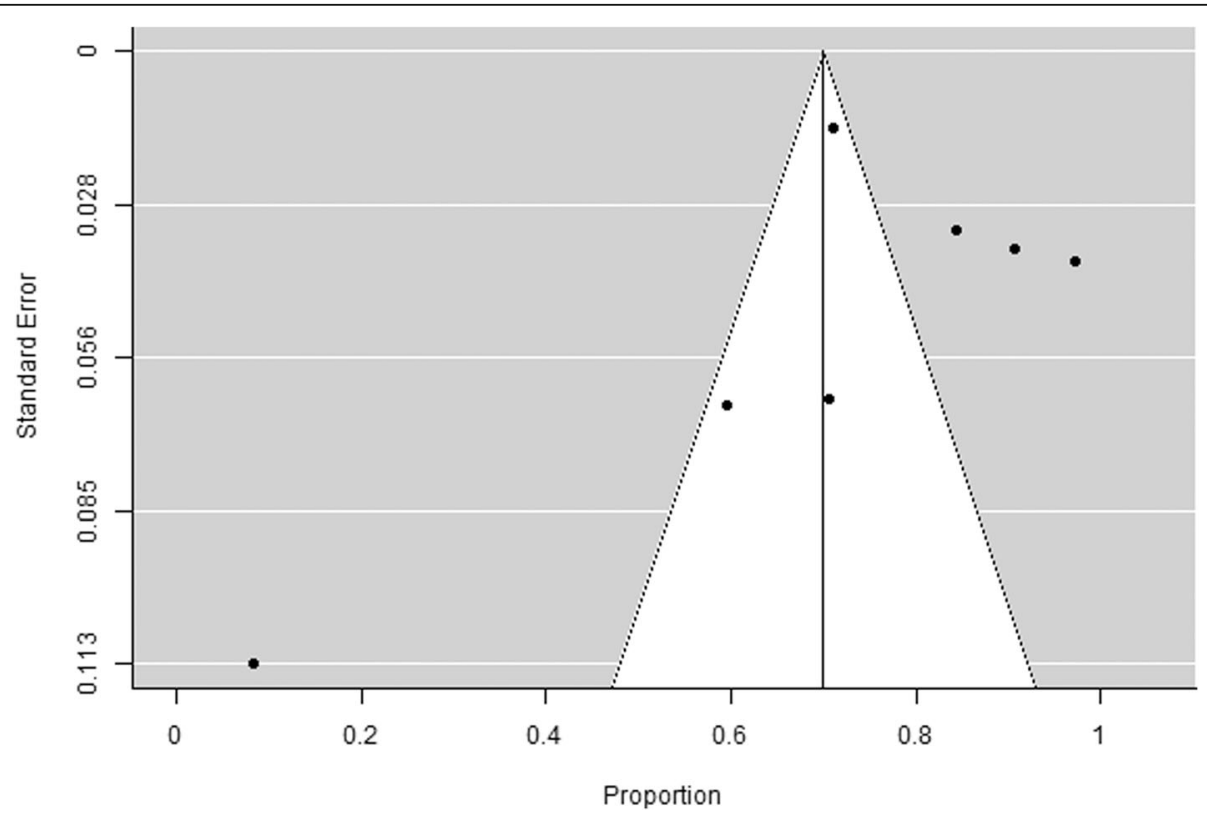

Fig. 4 Funnel plot for sensitivity of the initial CT scan. There is possibility of publication bias (Rosenthal fail-safe number $=7902$, rank correlation test for funnel plot asymmetry $P$ value $=0.562$, regression test for funnel plot asymmetry $P$ value $=0.002$ ) 
epidemiological point of view, however, CT analysis for early detection of SARS-CoV-2 infection needs to be done with caution [22].

On the other hand, Bernheim et al. stated that $56 \%$ of patients imaged early, (0-2) days from onset of symptoms, had normal CT appearance as compared to $9 \%$ of intermediate patients (3-5) days and $4 \%$ of late patients. So, early after symptom onset, chest CT has imperfect sensitivity and negative predictive value and is so unlikely a reliable standalone means to rule out COVID-19 infection [12]. Also, Chung et al. reported negative imaging in known infected patients COVID-19 at initial presentation and CT appearance of COVID-19 that shares similar findings with other diseases that cause viral pneumonia (3/21 patients) [20].

Another study reported a clashing finding that $7 / 19$ (37\%) asymptomatic cases, that had positive RT-PCR results, had no CT changes [23]. This data shows the limited value of CT screening in early COVID-19 diagnosis. Hence, we have sufficient reasons to question if CT is a suitable means for screening of asymptomatic infections. Additionally, studies have also shown that the 2ry attack rate among close contacts is $9.6 \%$ (95\% CI 7.9-11.8) [3], and that asymptomatic patients account for only around $1-2 \%$ of total SARS-CoV-2 infections [4].

The American College of Radiology (ACR) opposes the results of meta-analysis study and said that "CT chest should not be used to screen for or as a first-line for Covid-19 diagnostic test."

The ACR believes that the following factors considerations about the use of imaging for suspected or known COVID-19 infection:

- The Centers for Disease Control (CDC) currently does not recommend plain chest X-ray or CT to diagnose SARS-CoV-2 infection. Nucleic acid viral testing remains the only specific method of diagnosis. Even if COVID-19 radiologic findings are suggestive, confirmation with the viral test is required.

- Generally, chest X-ray or chest CT findings of COVID-19 are not specific and do overlap with findings seen in other infections, including influenza, H1N1, SARS, and MERS. The presence of a flu season with a much higher prevalence of influenza in the US than COVID-19, further limits the specificity of CT [24].

The Royal College of Radiologists announced that "there is no existing role for CT in the diagnostic assessment of patients with suspected infection with coronavirus in the UK." This was in March 12, 2020; however, in March 27, they said, chest CT assessment for the presence of likely COVID-19 infection might be beneficial in stratifying risk in acutely presenting patients. In the absence of quick access to COVID testing, this is suitable if it will affect the patient management. However, a negative CT scan result would not exclude COVID-19 infection. As with all other advice now, this may change [25].

The Royal Australian and New Zealand College of Radiology advised that "CT should not be used for routine screening for Covid-19 disease." [26].

The Canadian Association of Radiologists stated in March 26, 2020, that a normal chest CT scan cannot exclude COVID-19 particularly for patients with recent onset of symptoms. According to the Public Health Agency of Canada (PHAC) and the World Health Organization (WHO), the final diagnosis of COVID-19 infection should be confirmed by a positive RT-PCR test. This is standard of reference. The Canadian Society of Thoracic Radiology (CSTR)/Canadian Association of Radiologists (CAR) recommends against the use of routine chest CT for screening, diagnosis, and surveillance of COVID-19 infection. The CSTR/CAR recommends chest CT in patients with confirmed COVID-19 infection who may have acquired complications such as a lung abscess or empyema [27].

To date, most radiologic data has been coming from China. Some studies suggest that chest CT in the setting of a negative PCR test may be positive. Other studies deny the benefit of chest $\mathrm{CT}$ in the early diagnosis of COVID-19. All over the world, knowledge is still rapidly evolving and not all available information are published and or publicly shown. So, the possibility of using chest CT in early diagnosis of COVID-19 still needs more supportive data and also the use of chest CT in screening high-risk groups should be weighed against risks and benefits to reduce radiation dose. Clear criteria for the use of CT in diagnosis of COVID-19 should be established based on current available data. One criteria would be having symptoms or signs suggestive of infection or having contact with a diagnosed patient and have a positive RT-PCR test; another would be to treat or determine the course of the disease.

\section{Conclusion}

The meta-analysis study revealed that chest CT may be beneficial in early detection of cases of COVID-19. Further studies from different centers all over the world should be waited. Until that time imaging should be used as an adjunct to clinical and laboratory parameters in monitoring of disease course, until additional evidence is available.

\section{Abbreviations}

RT-PCR: Reverse transcriptase-polymerase chain reaction; CT: Computerized tomography; COVID-19: Coronavirus disease of 2019; CXR: Chest X-ray; SARSCoV-2: Severe acute respiratory syndrome coronavirus 2; PRISMA: Preferred Reporting Items for Systematic Reviews and Meta-Analyses 


\section{Acknowledgements}

We would like to express our great appreciation to Professor Magdy Amin Riad, Professor of Otolaryngology, Head and Neck Surgery at Ain Shams University Hospitals, Consultant ENT, Head and Neck Surgery, Ninewells Hospital and Medical School, Dundee, UK, for his valuable and constructive suggestions during this work. His willingness to give generously has been very much appreciated.

We would also like to offer our deepest thanks and gratitude to Professor Osama Hasan, Professor of Otolaryngology, Head and Neck Surgery at Ain Shams University Hospitals for his enthusiastic encouragement and useful critiques of this research work.

\section{Authors' contributions}

HM: performed data collection and shared in study selection. MT: proposed the idea, reviewed of literature, shared in data abstraction and manuscript writing. AA: was the corresponding author and shared in data abstraction. MA: performed the statistical analysis. AO: analyzed the included articles. SA: shared in study selection and data abstraction. All authors have read and approved the manuscript.

\section{Funding}

None to be mentioned.

\section{Availability of data and materials}

Available upon request

\section{Ethics approval and consent to participate}

Not applicable

\section{Consent for publication}

Not applicable as it is a systematic review

\section{Competing interests}

No conflict of interest to be declared

\section{Author details}

'Ear, Nose and Throat Department, Faculty of Medicine, Ain Shams University, Cairo, Egypt. ${ }^{2}$ Clinical Pathology Department, Al-Mataria Teaching Hospital, Cairo, Egypt. ${ }^{3}$ Clinical Pathology Department, Faculty of Medicine, Ain Shams University, Cairo, Egypt.

Received: 25 May 2020 Accepted: 24 September 2020

Published online: 09 November 2020

\section{References}

1. Kannan S, Shaik Syed Ali P, Sheeza A, Hemalatha K (2020) COVID-19 (novel coronavirus 2019) - recent trends. Eur Rev Med Pharmacol Sci 24(4):20062011

2. Li Q, Guan X, Wu P et al (2020) Early Transmission dynamics in Wuhan, China, of novel coronavirus-infected pneumonia. N Engl J Med

3. Guan WJ, Ni ZY, Hu Y et al (2020) Clinical characteristics of coronavirus disease 2019 in China. N Engl J Med

4. Chan JF, Yuan S, Kok KH et al (2020) A familial cluster of pneumonia associated with the 2019 novel coronavirus indicating person-to-person transmission: a study of a family cluster. Lancet 395:514

5. Huang C, Wang Y, Li X et al (2020) Clinical features of patients infected with 2019 novel coronavirus in Wuhan, China. Lancet 395:497

6. Chen N, Zhou M, Dong X et al (2020) Epidemiological and clinical characteristics of 99 cases of 2019 novel coronavirus pneumonia in Wuhan, China: a descriptive study. Lancet 395:507

7. Wang D, Hu B, Hu C et al (2020) Clinical characteristics of 138 hospitalized patients with 2019 novel coronavirus-infected pneumonia in Wuhan, China. JAMA

8. Pan F, Ye T, Sun P, et al. Time course of lung changes on chest $C T$ during recovery from 2019 novel coronavirus (COVID-19) pneumonia. Radiology 2020; :200370

9. Zhao W, Zhong Z, Xie X et al (2020) Relation between chest $C T$ findings and clinical conditions of coronavirus disease (COVID-19) pneumonia: a multicenter study. AJR Am J Roentgenol 1

10. Van Doremalen N, Bushmaker T, Morris DH et al (2020) Aerosol and surface stability of SARS-CoV-2 as compared with SARS-CoV-1. N Engl I Med
11. Ai T, Yang Z, Hou H, Zhan C, Chen C, Lv W, Xia L (2020) Correlation of chest CT and RT-PCR testing in coronavirus disease 2019 (COVID-19) in China: a report of 1014 cases. Radiology 200642

12. Bernheim A, Mei $X$, Huang M et al (2020) Chest CT findings in coronavirus disease-19 (COVID-19): relationship to duration of infection. Radiology 200463

13. Fang $Y$, Zhang $H$, Xie J, Lin M, Ying L, Pang P et al (2020) Sensitivity of Chest CT for COVID-19: comparison to RT-PCR. Radiology. 200432

14. Liji T (2020) Correlation of chest CT and RT-PCR testing in coronavirus disease 2019 (COVID-19) in China: a report of 1014 cases. News-Medical

15. Wong HYF, Lam HYS, Fong AH et al (2019) Frequency and distribution of chest radiographic findings in COVID-19 positive patients. Radiology. 201160 Epub 2019 Mar 27

16. Xie X, Zhong Z, Zhao W, Zheng C, Wang F, Liu J (2020) Chest CT for typical 2019-nCoV pneumonia: relationship to negative RT-PCR testing. Radiology. 200343

17. Li X, Zeng W, Li X et al (2020) CT imaging changes of corona virus disease 2019 (COVID-19): a multi-center study in Southwest China. J Transl Med 18:154

18. Xu G, Yang Y, Du Y et al (2020) Clinical pathway for early diagnosis of COVID-19: updates from experience to evidence-based practice. Clinic Rev Allerg Immunol 59:89-100

19. Center for Disease Control and Prevention. Interim guidelines for collecting, handling, and testing clinical specimens from persons under investigation (PUls) for coronavirus disease 2019 (COVID-19). 2020. https:/www.cdc.gov/ coronavirus/2019-ncov/lab/quidelines-clinical-specimens.html. Published February 14, 2020. Accessed February 28, 2020. Google Scholar

20. Kanne JP, Little BP, Chung JH, Elicker BM, Ketail LH (2020) Essentials for radiologists on COVID-19: an update-radiology scientific expert panel. Radiology

21. Chung M, Bernheim A, Mei X, Zhang N, Huang M, Zeng X, Cui J, Xu W, Zang Y, Fayad ZA, Jacobi A, Li K, Li S, Shan H (2020) CT imaging features of 2019 novel coronavirus (2019-nCoV). Radiology

22. Shi $H$, Han $\mathrm{X}$, Jiang $\mathrm{N}$ et al (2020) Radiological findings from 81 patients with COVID-19 pneumonia in Wuhan, China: a descriptive study. Lancet Infect Dis

23. Li J, Xu Q, Cuomo R, Purushothaman V, Mackey T (2020) Data mining and content analysis of the Chinese social media platform Weibo during the early COVID-19 outbreak: retrospective observational infoveillance study. JMIR Public Health Surveill 6(2):e18700

24. ACR Recommendations for the use of chest radiography and computed tomography (CT) for suspected COVID-19 infection. Cited MARCH 22, 2020.

25. RCR position on the role of $C T$ in patients suspected with COVID-19 infection [Internet]. Published on 12 March 2020. [cited 27 March 2020].

26. Royal Australian and New Zealand College of Radiology.COVID-19: position statements and guidance college position statements [Internet]. Advice on appropriate use of CT throughout the COVID-19 pandemic. [cited 9 April 2020].

27. Corrigendum to Canadian Association of Thoracic Radiology/Canadian Association of Radiologists Consensus Statement regarding chest imaging in suspected and confirmed COVID-19. Can Assoc Radiol J. 2020; 846537120931222. [Epub, 2020 May 18].

\section{Publisher's Note}

Springer Nature remains neutral with regard to jurisdictional claims in published maps and institutional affiliations. 